$\mathrm{Oz}$

Volume 30

Article 9

$1-1-2008$

\title{
Thoughts on Haptic Architecture By a Perceptual Psychologist
}

Howard C. Hughes

Follow this and additional works at: https://newprairiepress.org/oz

(c) (i) $\Theta \Theta$

This work is licensed under a Creative Commons Attribution-Noncommercial-No Derivative Works 4.0 License.

\section{Recommended Citation}

Hughes, Howard C. (2008) "Thoughts on Haptic Architecture By a Perceptual Psychologist," Oz: Vol. 30. https://doi.org/10.4148/2378-5853.1458

This Article is brought to you for free and open access by New Prairie Press. It has been accepted for inclusion in Oz by an authorized administrator of New Prairie Press. For more information, please contact cads@k-state.edu. 


\title{
Thoughts on Haptic Architecture \\ By a Perceptual Psychologist
}

\author{
Howard C. Hughes
}

Like most people, I do not know much about architecture, but I spend a great deal of time in buildings. I was therefore both intrigued and a little intimidated when I was asked by the editors of $\mathrm{Oz}$ to write an article on "haptic architecture." In expressing my thoughts and reactions, I will tread lightly and with some trepidation, because I am not accustomed to addressing topics on which, unlike my audience, I have no particular expertise.

Let us begin by considering the use of the word "haptic" in haptic architecture. Reference to any dictionary tells us that haptic refers to the sense of touch. For perceptual psychologists, it usually refers to active touch - a form of exploratory touching in which the hands palpate an object in order to get the richest possible representation using touch alone. Used in this sense, the haptic system is really a multimodal system that combines information from cutaneous receptors (a variety of sensory receptors in our skin) with information from our kinesthetic system, which provides information about our posture, movements, and effect of external forces like gravity and the masses of objects we manipulate. However, it seems that the proponents of haptic architecture have something more in mind. Something akin to a characterization of haptic perception includes:

the sensing offabrics by the hand; the sensing offood textures by the mouth; the sensing of vibrations in machinery that signify normal or abnormal objects and their spatial arrangement; the sensing of imperfections and dirt on the surfaces of objects; the examina tion of internal organs of the body by palpation..., and the moment of inertia of hefted objects...For humans...it contributes much to social and sexual communication, to individualdevelop ment, and to the aesthetic appreciation of both art and daily life. (Loomis and Lederman, 1986).

If this is what is meant, haptic architecture has indeed set a lofty goal for itself. According to Pallasmaa (2000), modern architecture suffers from the "hegemony of vision," which he appears to attribute to a failing of modern society, and perhaps a failing of modern architects to resist succumbing to society's failure. Thus, he writes

Ibelieve likewise that many aspects of the pathology of today's architecture can also be understood through a critique of the ocular bias of our culture. As a consequence of the power of the eye over the other sensory realms, architecture has turned into an art form of instant visual image. Instead of creating existential microcosms, embodied representations of the world, architecture projects retinal images for the purpose of immediate persuasion. Flatness of surfaces and materials, uniformity of illumination, as well as the elimination of microclimatic differences, further reinforce the tiresome and soporific uniformity of experience." (Pallasmaa, 2000).

I could not agree more with the assertion that the "flatness of surfaces and materials, the uniformity of illumination" and other salient aspects of many modern architectural works leads to a "tiresome and soporific uniformity of experience." However, I do not believe that any architecturally-induced stupor is properly attributed to the "hegemony of vision." It is, I think, more appropriately attributable to a failure of architectural designs to provide the richness of color, texture, form and depth that resonate with the mechanisms of human vision. No one who studies human perception would consider, much less lament, the "he-

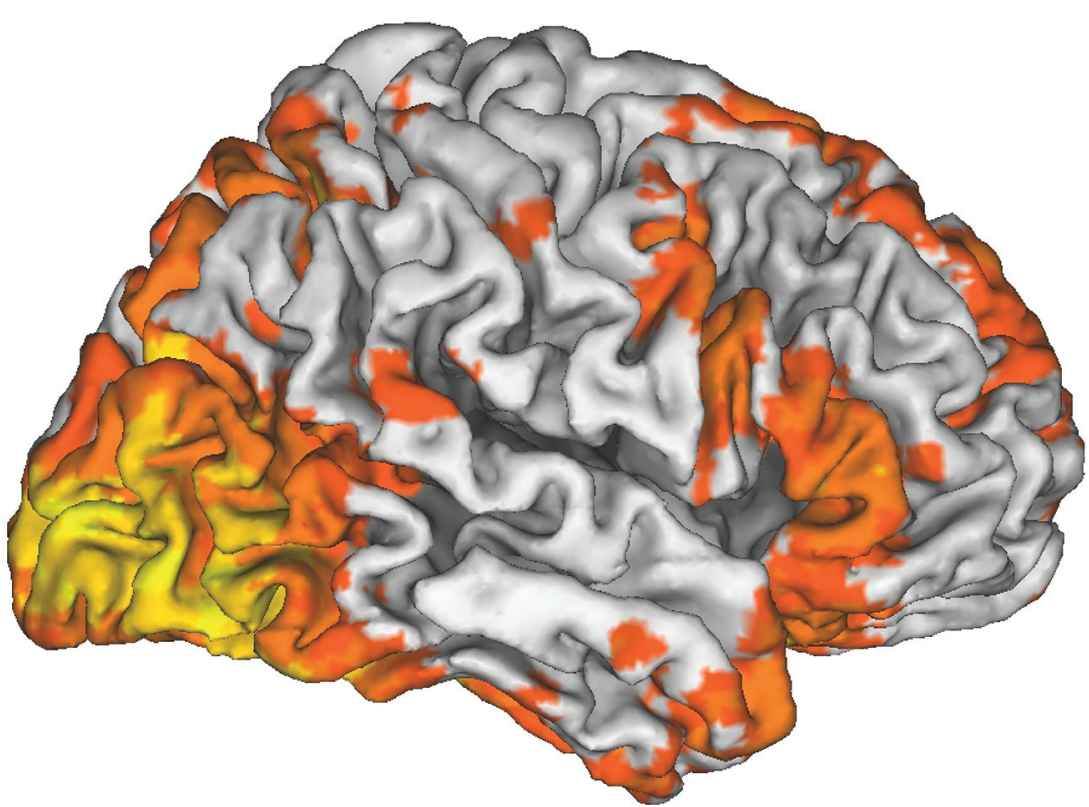

Figure 1. This illustrates the pattern of neural activity within the right cerebral cortex evoked by passive viewing of a series of visual scenes. Yellow areas indicate regions of greatest activity, red areas indicate areas of lesser (but still statistically significant) neural activations. A similar pattern appears in the left hemisphere (not shown). This is actually a statistical map obtained from eight observers. As such, it represents the intersection of activations in all eight people. This is a conservative measure, as each individual person's activity profile would actually be more extensive than what is shown here. These neural activations were obtained using functional magnetic resonance imaging techniques at the Brain Imaging Center in the Department of Psychological and Brain Science at Dartmouth College. Photo courtesy of Prof. William Kelley and Dr. Kathryn Demos. gemony" of vision. The preeminence of vision is not an accident or a wrong turn taken during the development choice that we can choose make ( 200,000 years of human evolution. As such, it is much better to understand the power of human visual perception than to view it as a regrettable mistake that should somehow be corrected. I suggest that the solution to any perceived ills in modern architecture is not to resist the dominance of vision operation; the identification of solid 
over other modalities, but to accept that dominance and simply make our buildings more pleasing and more attuned to the fundamental properties of the incredibly intricate system of neuronal circuits that mediates our sense of visual perception. Perhaps if architects had a better understanding of human vision, they would celebrate the hegemony of vision, rather than resist what is really irresistible.

With those initial thoughts in mind let us briefly turn our attention to one of the most astonishing, complex and exquisite devices in the known universe-the human visual system. When you look at a picture of a human brain, the first thing that captures your attention is that the outer surface is all wrinkled. The outer surface of the brain is composed of what is called the cerebral cortex, and those wrinkles in the cerebral cortex are called convolutions. The surface area of the cerebral cortex is approximately 2.64 square feet. The cortex is so heavily convoluted because that is the only way to fit such a large sheet of neural tissue into a skull that is a little more than 7 inches wide (despite its large surface area, the cortex is only about 0.1 inches thick). Not all mammals have such a heavily convoluted cortex, however. The cortex of rodents is completely smooth, and that of cats and dogs is very sparsely convoluted. During the course of human evolution, the cerebral cortex has undergone a dramatic expansion relative to other regions of the central nervous system, and that is why our cortex is more heavily convoluted than virtually any other creature (dolphins are our only rival in this respect). The cerebral cortex is intimately involved in vision, hearing touch, memory, language, thought and consciousness. As such, the cerebra cortex is essential to all the menta processes that make us human. That is why the functions of the cerebral cortex hold so much fascination for all psychologists.

As the proud owner of the most intricate cerebral cortex in the known universe, you may be interested to know that neuroscientists estimate that over $50 \%$ of that magnificent neural structure is involved in vision. This is well-illustrated in Figure 1, which shows the pattern of cortical activity evoked while human observers passively viewed a sequence of still images (familiar objects, buildings landscapes, and other people). Note that this widespread activity is generated by passive viewin-it does not include additional brain activations that would be recruited if you were actively searching for a particular item in the scenes, remembering them for later recall, or attempting to interact with the images in any way. This fairly dramatic example illustrates how much of the human brain is devoted to vision. No other sensory modality could evoke so much brain activity with so little corresponding mental effort. Figure 1 makes it clear that ascendancy of vision over all other sensory modalities is not something that architects can ignore. Rather, it is an essential part of our nature. I assume that everyone agrees that architecture is one of the visual arts, so the challenge is to design buildings that resonate with our very elaborate visual mechanisms.

My sense is that many of the trends in modern architecture fail to provide the features the human visual system is designed to process. As a result, a great deal of modern architecture provides the perceptual system with a highly impoverished environment. I accept that Mr. Pallasmaa (2000) is largely correct in stating that prevalence of flat surfaces, the lack of color contrast, the use of uniform materials that result in uniform textures and unvarying lightning are by and large accurate. do not know why the architecture of the later part of the twentieth century is so lacking in adornment. The human visual system is "tuned" to respond to contrast and change, and to purposely avoid changes in color, shading, and texture in our architectural designs is to deny our perceptual systems the essential elements of the visual world they are designed to convey.

The visual system appears to have evolved in such a manner as to be especially sensitive to certain statistica properties that occur in natural scenes (Hughes, Nozawa and Kitterle, 1996). Natural materials such as stone, bark, wood grain, and water share a deep similarity in their image structure, and our perceptual system contains mechanisms that are specifically designed to "resonate" with those natural textures. These regularities are the same as those embodied in the geometry of fractals (Mandelbrot, 1982). Fractals have a type of irregularity called "self-similarity," which means that the geometric structure of fractals recapitulates itself at multiple spatial scales. For instance, when you look at a snow flake, the articulated pattern of branching ice crystals is very salient. If you were to look at a small portion of the snow flake under a microscope, that pattern would be evident at a finer scale. If you increased the magnification, that pattern would again reappear at a still finer spatial scale. This is the property of self-similarity. Fractals are ubiquitous in nature. Besides snowflakes, other well-known natural examples are mountains, coastlines, trees, and the shapes of neurons. Uniform textures of smooth concrete and glass will not have these properties. I think some of the awe and timeless beauty of Frank Lloyd Wright's masterpiece Fallingwater may derive from his inspired use of natural textures (perhaps in judicious juxtaposition with some carefully chosen man-made materials). Our sense of vision is tuned to and "resonates" with those natural textures.

"Hapticity" implies integration from different sensory modalities, so let us now turn to a brief consideration of multi-sensory interactions and integration, a topic that has received a great deal of attention in sensory physiology and perceptual science. The problem from a scientific perspective can be concisely described as follows: if each sensory modality (vision, audition, touch) is conveyed to the central nervous system over separate, modality-specific neural pathways, how can we explain the phenomenological unification of our perceptual experience? Our experience 


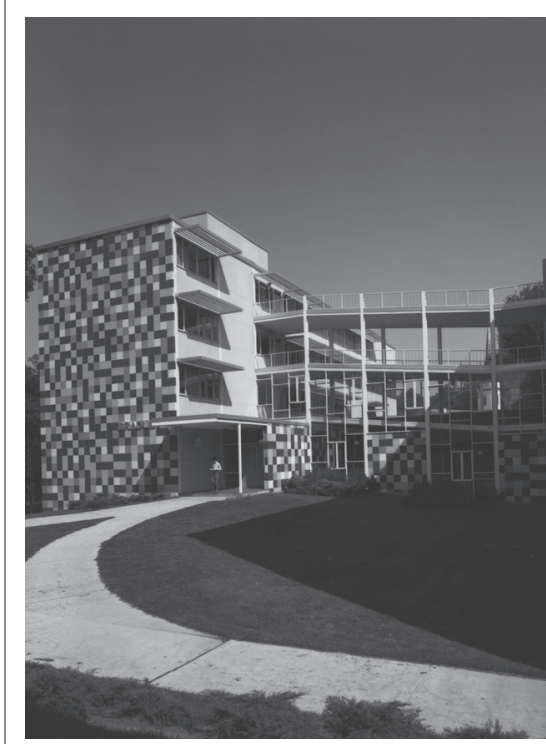

Figure 2. A photo of Gerry Hall (circa 1967), for

is not one of separate visual, auditory, and tactile sensations. We can tell how a surface will feel simply by looking at it. We can tell what an unseen object will look like through active touch (but not nearly so much through passive touch). We hear running water, and immediately know how swiftly it flows, the height of the fountain, or the volume of water involved. This is called multimodal integration, and there is good evidence that integration of the senses is mediated by highly specific patterns of convergence of modality-specific neural pathways that create polymodal centers in the brain These patterns of convergence are such that congruent information arising from different modalities is usually mutually reinforcing, but incongruent information causes conflict and mer home of the Department of Psychology at Dartmouth College. The tiles were various shades ofblue. The remaining walls were clad in granite slabs. The interior walls were all a medium shade of brown ceramic tile. It was not a good environment for working or teaching. It is reputed that the architect was a former Dartmouth student Although Dartmouth is famed for its loyal and generous alumni, the speculation was that this particular student was not quite as happy as most. Photograph courtesy of Patricia B Cope Photographic Records Specialist, Special Collections, Dartmouth College.

not counter the effect of the goggles and make a straight edge look straight If there is a lesson that perceptual science can offer architects with respect to a multimodal haptic architecture, it probably is to work toward designs in which the senses of touch and hear ing are consistent with and reinforce (without replacing or conflicting with) the visual experience of architectural spaces. The sounds of falling water are soothing, but if those sounds are made artificial by hearing them in a large atrium in which all the glass and metal surfaces produce multiple echoes, much of the aesthetic potential may be lost, as the sound then seems artificial and inconsistent with what is seen (we are actually quite good at processing echoes, and they don't need to be of the obvious sort you hear when you shout in a canyon). If materials rich in texture are used because they are pleasing to the eye, designs might be attempted in which the visitor is somehow invited to contact those materials directly (perhaps by clever uses in hand rails used in stairs and corridors) tively straight edge will "feel" straight even if the eyes are closed. Similarly, palpation of a curved edge will feel curved. However, if the information conveyed by vision and active touch is placed into conflict, then the visual cues usually dominate. Thus, after a prolonged period of wearing special goggles that optically distort the seen world such that straight edges appear curved, the visual distortion results in changes in haptic touch, and straight contours come to feel curved! Note that the reverse adaptation does not occur-feeling the straight edge does makes you pause and think to yourself, "what were they thinking when they built that monstrosity!" On the campus of Dartmouth College, the buildings that no one liked were the home of the Departments of Psychology and Mathematics. The architect was an alumnus of Dartmouth College (I always wondered whether this individual got poor grades in Psychology or Mathematics, and was therefore disaffected with our departments). The Gerry-Bradley complex consisted of twin buildings (yes, astonishingly they built two of them!). The Psychology department was in Gerry Hall, and Mathematics was in Gerry's twin, Bradley Hall. Each had a wall that was tiled in shades of blue. During the 1970s (before my time here), a popular student prank was to climb to the roof and hang a gigantic fake shower head over the top of the tiled wall. Gerry Hall thus became known as the "shower tower." The entire interior of both buildings were lined with a medium to dark shade of brown ceramic tile. Perhaps you can imagine the adjectives that were used to describe the exact shade of brown. There was plenty of glass in the building too. The problem, however, was that it was all single-pane glass. Large single-pane windows in New Hampshire are not a good idea. During the bitter cold winter months, the cold air used to fall from those glass windows like the Niagara River cascading over the falls. In my office, which was strategically placed on the north side of Gerry Hall, this flow of cold air would actually rustle all the papers attached to the bulletin board above my desk. In point of fact, there 
wasn't a single redeeming feature of Gerry Hall, and we were all happy to see it demolished in 2007.

I think the time I served in Gerry Hall ("hard time" is what I'd like to call it) entitles me to the following closing thoughts, which I offer in the spirit of constructive advice (and hope). First, despite whatever intellectual analyses one might make, architecture is a visual art. The primary experience of a building is a visual experience, so we should strive to make that experience as pleasing as possible. Secondly, although architecture is a visual art, it is very different from the other visual arts. A painter or sculptor has no real obligation to society in general, because people are not compelled to visit or view their work. Buildings are very different. Frequently people have no choice-they must pass the building, and may even have to work in it. It seems to me that this simple fact means that architects have a responsibility to society that painters or sculptors usually do not. Mr. Pallasmaa (2000) is correct: don't just think about immediate visual impression a building might provide. Always bear in mind that many people, people who you will never know and do not approach building design as an intellectual exercise, will likely spend significant amounts of time in the spaces you designed. Finally, perhaps architects should receive at least a little training in the mechanisms of visual perception. If the designer of Gerry Hall had taken Perception as a Dartmouth student, perhaps the building would still be standing.

\section{References}

Hughes, H.C. Nozawa, G. \& Kitterle, F "Global precedence, spatial frequency channels, and the statistics of natural images," Journal of Cognitive Neuroscience, Vol. 8, pp. 197-230, 1996.

Loomis, J.M. \& Lederman, S.J. “Tactual perception." In K.R. Boff, L. Kaufman \& J.P. Thomas (Eds.). Handbook of perception and human performance. Volume II: Cognitive processes and performance. New York: John Wiley, 1986.

Mandelbrot, Benoît. The Fractal Geometry of Nature, W. H. Freeman \& Co., 1982.

Pallasmaa,J. “Hapticity and Time - discussion of haptic, sensuous architecture. The Architectural Review, May, 2000.

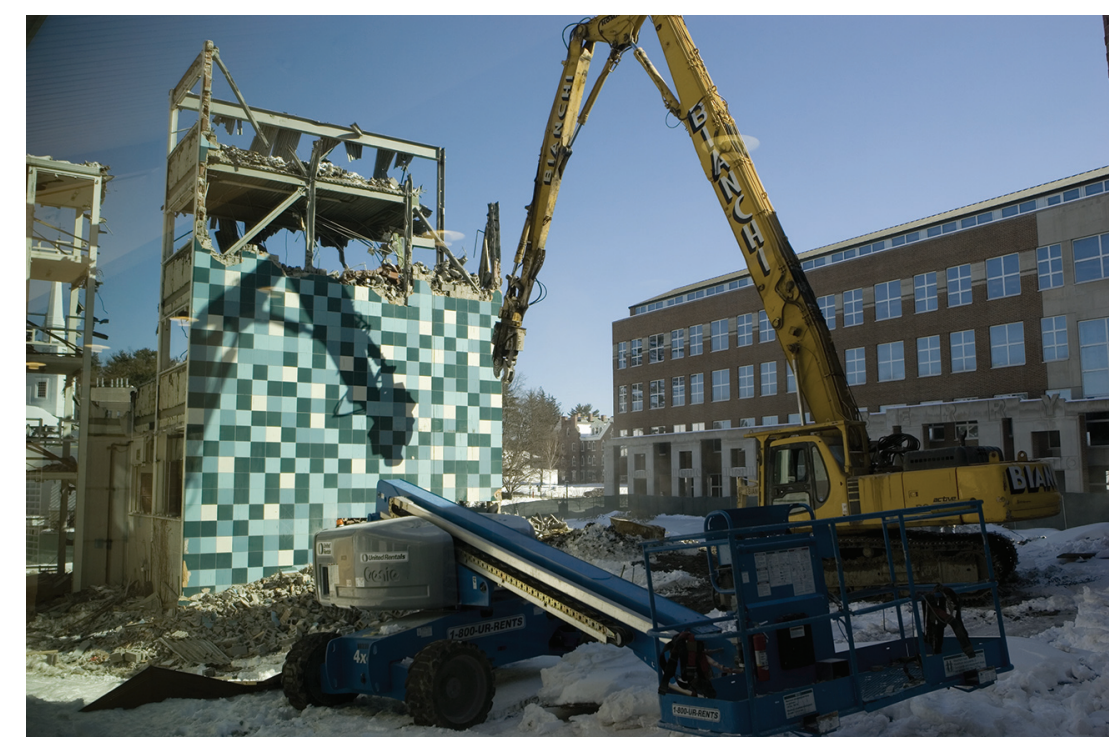

Figure 3. Gerry-Bradley Hall in January, 2007. Photograph courtesy of Joseph Mehling, Dartmouth College Photographer 